\title{
Correction to: Generalized similarity measures under linguistic $q$-rung orthopair fuzzy environment with application to multiple attribute decision-making
}

\author{
Rajkumar Verma ${ }^{1}$
}

Published online: 16 July 2021

(C) Springer Nature Switzerland AG 2021

\section{Correction to: Granular Computing https://doi.org/10.1007/s41066-021-00264-4}

In this article several suffix values were missed and some of them were incorrect in the numerical examples while processing. The corrected statements are provided below.

1. The corrected Definitions 2 and 3 are provided below:

Definition 2 Let $\mathfrak{U}=\left\{u_{1}, u_{2}, \ldots, u_{n}\right\}$ be a fixed set and $\widehat{\mathbb{L}}_{[0,2 t]}=\left\{\ell_{d} \mid \ell_{0} \leq \ell_{d} \leq \ell_{2 t}, d \in[0,2 t]\right\}$ be an extended continuous LTS, then a $\mathrm{L}^{q}$ ROPFS in $\mathfrak{U}$ can be defined as

$3=\left\{\left\langle u, \ell_{\xi_{3}(u)}, \ell_{\eta_{3}(u)}\right\rangle \mid u \in \mathfrak{U}\right\}$

where

$$
\ell_{\xi_{3}(u)}, \ell_{\eta_{3}(u)} \in \widehat{\mathbb{L}}_{[0,2 t]},
$$

satisfying $0 \leq\left(\xi_{3}(u)\right)^{q}+\left(\eta_{3}(u)\right)^{q} \leq(2 t)^{q} \forall u \in \mathfrak{U}$ with $q \geq 1$. The LTs $\ell_{\xi_{3}(u)}$ and $\ell_{\eta_{3}(u)}$ represent, respectively, the DM and DNM of an element $u \in \mathfrak{U}$ to the $\mathrm{L}^{q}$ ROPFS 3 . The degree of hesitancy of $u \in \mathfrak{U}$ to the set 3 can be calculated as $\ell_{\psi_{3}(u)}=\ell_{\sqrt[q]{(2 t)^{q}-\left(\xi_{3}(u)\right)^{q}-\left(\eta_{3}(u)\right)^{q}}}$. For a given element $u \in \mathfrak{U}$, the pair $\left\langle\ell_{\xi_{3}(u)}, \ell_{\eta_{3}(u)}\right\rangle$ is called a $\mathrm{L}^{q} \mathrm{ROPFN}$, which can be simply denoted by $\Upsilon=\left\langle\ell_{\xi_{\Upsilon}}, \ell_{\eta_{\Upsilon}}\right\rangle$.

Definition 3 (Liu and Liu 2018b) Let $3_{1}$ and $3_{2}$ be two $\mathrm{L}^{q}$ ROPFSs in $\mathfrak{U}$, then

i $\quad 3_{1} \subseteq 3_{2}$ if and only if $\ell_{\xi_{3_{1}}}(u) \leq \ell_{\xi_{3_{2}}}(u)$ and $\ell_{\eta_{3_{1}}(u)} \geq \ell_{\eta_{3_{2}}(u)} \forall u \in \mathfrak{U}$;

ii $\boldsymbol{3}_{1}=\boldsymbol{3}_{2}$ if and only if $\boldsymbol{3}_{1} \subseteq \boldsymbol{3}_{2}$ and $\boldsymbol{3}_{1} \supseteq \boldsymbol{3}_{2}$;

The original article can be found online at https:// doi.org/10.1007/s41066-021-00264-4.

Rajkumar Verma

rkver83@gmail.com; rverma@fen.uchile.cl

Department of Management Control and Information Systems, University of Chile, Av. Diagonal Paraguay 257, 8330015 Santiago, Chile

$$
\begin{aligned}
& \text { iii } \left.\quad 3_{1}^{C}=\left\{\left\langle u, \ell_{\eta_{3_{1}}(u)}, \ell_{\xi_{3_{1}}(u)}\right\rangle\right\rangle \mid u \in \mathfrak{U}\right\} \\
& \text { iv } 3_{1} \bigcup^{q} 3_{2}=\left\{\left\langleu, \max \left(\ell_{\xi_{3_{1}}}(u), \ell_{\xi_{3_{2}}(u)}\right)\right.\right. \text {, } \\
& \left.\left.\min \left(\ell_{\eta_{3_{1}}(u)}, \ell_{\eta_{3_{2}}(u)}\right)\right\rangle \mid u \in \mathfrak{U}\right\} ; \\
& \mathrm{v} \quad 3_{1} \bigcap^{q} 3_{2}=\left\{\left\langleu, \min \left(\ell_{\xi_{3_{1}}(u)}, \ell_{\xi_{3_{2}}(u)}\right)\right.\right. \text {, } \\
& \left.\left.\max \left(\ell_{\eta_{3_{1}}(u)}, \ell_{\eta_{3_{2}}(u)}\right)\right\rangle \mid u \in \mathfrak{U}\right\} \\
& \text { vi } 3_{1} \stackrel{q}{\oplus} 3_{2}= \\
& \left\{\left\langle u, \ell \underset{q}{2 t \sqrt[q]{\frac{\xi^{q}{ }_{1}(u)}{(2 t)^{q}}+\frac{\xi^{q}(u)}{(2 t)^{q}}-\frac{\xi^{q}(u) \xi_{32}(u)^{q}}{(2 t)^{q}}(2 t)^{q}}}, \ell_{2 t\left(\frac{\eta_{31}(u) \eta_{32}(u)}{(2 t)^{2}}\right)}\right\rangle\right. \\
& \mid u \in \mathfrak{U}\} \text {; } \\
& \text { vii } 3_{1} \stackrel{q}{\otimes} \mathfrak{3}_{2}=
\end{aligned}
$$

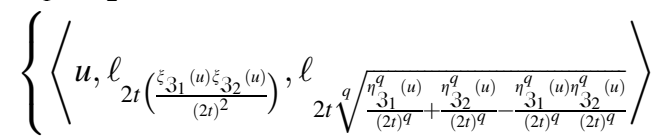

$$
\begin{aligned}
& \mid u \in \mathfrak{U}\} \text {. }
\end{aligned}
$$

2. The third point in Example 1 is as follows:

Example 1 Let $\widehat{\mathbb{L}}_{[0,6]}=\left\{\ell_{d} \mid d \in[0,6]\right\}$ be a continuous LTS, then the inverse corresponding to the LSFs $\Phi_{1}^{*}, \Phi_{2}^{*}$ and $\Phi_{3}^{*}$ can be obtained as follows:

1. $\Phi_{1}^{*}\left(\ell_{d}\right)=\kappa_{d}=\frac{d}{6}, d=[0,6], \quad$ then $\Phi_{1}^{*-1}\left(\kappa_{d}\right)=\ell_{6 \times \kappa_{d}}\left(\kappa_{d} \in[0,1]\right)$.

2. $\Phi_{2}^{*}\left(\ell_{d}\right)=\kappa_{d}=\left\{\begin{array}{ll}\frac{\varrho^{3}-\varrho^{3-d}}{2 \times\left(\varrho^{3}-1\right)}, & d=0 \leq d \leq 3 \\ \frac{\varrho^{3}+\varrho^{d-3}-2}{2 \times\left(\varrho^{3}-1\right)} & d=3<d \leq 6,\end{array}\right.$ then

$$
\begin{gathered}
\Phi_{2}^{*-1}\left(\kappa_{d}\right)= \\
\ell_{d}=\left\{\begin{array}{cc}
\ell_{3-\log _{\varrho}\left[\varrho^{3}-\left(2 \varrho^{3}-2\right) \kappa_{d}\right],} & \kappa_{d} \in[0,0.5] \\
\ell_{3+\log _{\varrho}\left[\left(2 \varrho^{3}-2\right) \kappa_{d}-\varrho^{3}+2\right]} & \kappa_{d} \in(0.5,1]
\end{array} .\right.
\end{gathered}
$$

3. $\Phi_{3}^{*}\left(\ell_{d}\right)=\kappa_{d}= \begin{cases}\frac{3^{\rho}-(3-d)^{\rho}}{2 \times 3^{\rho}}, & d=0 \leq d \leq 3 \\ \frac{3^{\tau}+(d-3)^{\tau}}{2 \times 3^{\tau}} & d=3<d \leq 6,\end{cases}$ 
then

$$
\Phi_{3}^{*-1}\left(\kappa_{d}\right)=\ell_{d}=\left\{\begin{array}{ll}
\ell_{3-\left[3^{\rho}-2 \times 3^{\rho} \times \kappa_{d}\right]^{\frac{1}{\rho}}}, & \kappa_{d} \in[0,0.5] \\
\ell_{3+\left[2 \times 3^{\tau} \times \kappa_{d}-3^{\tau}\right]^{\frac{1}{\tau}}} & \kappa_{d} \in(0.5,1]
\end{array} .\right.
$$

3. The corrected SP3 in Theorem 1 is as follows: SP3: Let $\widehat{S_{1}}\left(3_{1}, 3_{2}\right)=1$, that is,

$$
\begin{aligned}
& \sum_{j=1}^{n} \cos \left[\left\{\left|\left(\Phi^{*}\left(\ell_{\xi_{3_{1}}\left(u_{j}\right)}\right)\right)^{q}-\left(\Phi^{*}\left(\ell_{\xi_{3_{2}}\left(u_{j}\right)}\right)\right)^{q}\right|^{\lambda}\right.\right. \\
& +\left|\left(\Phi^{*}\left(\ell_{\eta_{3_{1}}\left(u_{j}\right)}\right)\right)^{q}-\left(\Phi^{*}\left(\ell_{\eta_{3_{2}}\left(u_{j}\right)}\right)\right)^{q}\right|^{\lambda} \\
& \left.\left.\quad+\left|\left(\Phi^{*}\left(\ell_{\psi_{3_{1}}\left(u_{j}\right)}\right)\right)^{q}-\left(\Phi^{*}\left(\ell_{\psi_{3_{2}}\left(u_{j}\right)}\right)\right)^{q}\right|^{\lambda}\right\}^{\frac{1}{\lambda}} \frac{\pi}{2^{\frac{1+\lambda}{\lambda}}}\right]=n,
\end{aligned}
$$

or

$$
\begin{aligned}
\cos & {\left[\left\{\left|\left(\Phi^{*}\left(\ell_{\xi_{3_{1}}\left(u_{j}\right)}\right)\right)^{q}-\left(\Phi^{*}\left(\ell_{\xi_{3_{2}}\left(u_{j}\right)}\right)\right)^{q}\right|^{\lambda}\right.\right.} \\
+ & \left|\left(\Phi^{*}\left(\ell_{\eta_{3_{1}}\left(u_{j}\right)}\right)\right)^{q}-\left(\Phi^{*}\left(\ell_{\eta_{3_{2}}\left(u_{j}\right)}\right)\right)^{q}\right|^{\lambda} \\
+ & \left.\left.\left|\left(\Phi^{*}\left(\ell_{\psi_{3_{1}}\left(u_{j}\right)}\right)\right)^{q}-\left(\Phi^{*}\left(\ell_{\psi_{3_{2}}\left(u_{j}\right)}\right)\right)^{q}\right|^{\lambda}\right\}^{\frac{1}{\lambda}} \frac{\pi}{2^{\frac{1+\lambda}{\lambda}}}\right]=1, \\
& \forall u_{j} \in \mathfrak{U}
\end{aligned}
$$

or

$$
\begin{aligned}
& \left\{\left|\left(\Phi^{*}\left(\ell_{\xi_{3_{1}}\left(u_{j}\right)}\right)\right)^{q}-\left(\Phi^{*}\left(\ell_{\xi_{3_{2}}\left(u_{j}\right)}\right)\right)^{q}\right|^{\lambda}\right. \\
& \quad+\left|\left(\Phi^{*}\left(\ell_{\eta_{3_{1}}\left(u_{j}\right)}\right)\right)^{q}-\left(\Phi^{*}\left(\ell_{\eta_{3_{2}}\left(u_{j}\right)}\right)\right)^{q}\right|^{\lambda} \\
& \left.\quad+\left|\left(\Phi^{*}\left(\ell_{\psi_{3_{1}}\left(u_{j}\right)}\right)\right)^{q}-\left(\Phi^{*}\left(\ell_{\psi_{3_{2}}\left(u_{j}\right)}\right)\right)^{q}\right|^{\lambda}\right\}^{\frac{1}{\lambda}}=0,
\end{aligned}
$$

$\forall u_{j} \in \mathfrak{U}$.

Since $\lambda \geq 1$, then Eq. (11) gives

$$
\begin{aligned}
& \left(\Phi^{*}\left(\ell_{\xi_{3_{1}}\left(u_{j}\right)}\right)\right)^{q}=\left(\Phi^{*}\left(\ell_{\xi_{3_{2}}\left(u_{j}\right)}\right)\right)^{q}, \\
& \quad\left(\Phi^{*}\left(\ell_{\eta_{3_{1}}\left(u_{j}\right)}\right)\right)^{q}=\left(\Phi^{*}\left(\ell_{\eta_{3_{2}}\left(u_{j}\right)}\right)\right)^{q}, \\
& \quad\left(\Phi^{*}\left(\ell_{\psi_{3_{1}}\left(u_{j}\right)}\right)\right)^{q}=\left(\Phi^{*}\left(\ell_{\psi_{3_{2}}\left(u_{j}\right)}\right)\right)^{q}, \quad \forall u_{j} \in \mathfrak{H} .
\end{aligned}
$$

which implies

$$
\begin{gathered}
\ell_{\xi_{3_{1}}\left(u_{j}\right)}=\ell_{\xi_{3_{2}}\left(u_{j}\right)}, \ell_{\eta_{3_{1}}\left(u_{j}\right)}=\ell_{\eta_{3_{2}}\left(u_{j}\right)}, \\
\ell_{\psi_{3_{1}}\left(u_{j}\right)}=\ell_{\psi_{3_{2}}\left(u_{j}\right) \quad u_{j} \in \mathfrak{U} .}
\end{gathered}
$$

Again consider $\mathfrak{Z}_{1}=\mathfrak{3}_{2}$, which means

$$
\begin{aligned}
& \left|\left(\Phi^{*}\left(\ell_{\xi_{3_{1}}\left(u_{j}\right)}\right)\right)^{q}-\left(\Phi^{*}\left(\ell_{\xi_{3_{2}}\left(u_{j}\right)}\right)\right)^{q}\right|=0, \\
& \left|\left(\Phi^{*}\left(\ell_{\eta_{3_{1}}\left(u_{j}\right)}\right)\right)^{q}-\left(\Phi^{*}\left(\ell_{\eta_{3_{2}}\left(u_{j}\right)}\right)\right)^{q}\right|=0, \\
& \left|\left(\Phi^{*}\left(\ell_{\psi_{3_{1}}\left(u_{j}\right)}\right)\right)^{q}-\left(\Phi^{*}\left(\ell_{\psi_{3_{2}}\left(u_{j}\right)}\right)\right)^{q}\right|=0, \quad \forall u_{j} \in \mathfrak{U} .
\end{aligned}
$$

Therefore, we get $\widehat{S_{1}}\left(\mathbf{3}_{1}, \mathbf{3}_{2}\right)=1$.

4. The corrected Theorem 3 is as follows:

Theorem 3 For $3_{1}, 3_{2}, 3_{3} \in \mathrm{L}^{\mathrm{q}} \mathrm{ROPFS}(\mathfrak{U})$, the following properties are true

(i) $\begin{aligned} & \widehat{S}_{k}\left(\mathcal{Z}, 3^{C}\right)=1 \quad \text { if } \quad \text { and } \text { only if } \\ & \ell_{\xi_{3}\left(u_{j}\right)}=\ell_{\eta_{3}\left(u_{j}\right)} \forall u_{j} \in \mathfrak{U} ;\end{aligned}$

(ii) $\stackrel{\omega}{\widehat{S}_{k}}\left(3_{1} \bigcup^{q} 3_{2}, 3_{1} \bigcap^{q} 3_{2}\right)=\stackrel{\omega}{S_{k}}\left(3_{1}, 3_{2}\right)$;

(iii) $\stackrel{\omega}{S_{k}}\left(3_{1}, 3_{1} \bigcap^{q} 3_{2}\right)=\stackrel{\omega}{S_{k}}\left(3_{2}, 3_{1} \bigcup^{q} 3_{2}\right)$;

(iv) $\hat{S}_{k}\left(3_{1}, 3_{1} \bigcup^{q} 3_{2}\right)=\stackrel{\omega}{S_{k}}\left(3_{2}, 3_{1} \bigcap^{q} 3_{2}\right)$;

(v) $\stackrel{\omega}{\widehat{S}_{k}}\left(3_{1} \cup^{q} 3_{2}, 3_{3}\right) \leq \stackrel{\omega}{\widehat{S}_{k}}\left(3_{1}, 3_{3}\right)+\stackrel{\omega}{\widehat{S}_{k}}\left(3_{2}, 3_{3}\right)$;

(vi) $\stackrel{\omega}{\widehat{S}_{k}}\left(3_{1} \bigcap^{q} \boldsymbol{3}_{2}, 3_{3}\right) \leq \stackrel{\omega}{S_{k}}\left(3_{1}, 3_{3}\right)+\stackrel{\omega}{\widehat{S}_{k}}\left(3_{2}, 3_{3}\right)$;

(vii) $\stackrel{\omega}{S_{k}}\left(3_{1} \bigcup^{q} 3_{2}, 3_{3}\right)+\stackrel{\omega}{S_{k}}\left(3_{1} \bigcap^{q} 3_{2}, 3_{3}\right)$

$$
=\widehat{S}_{k}\left(3_{1}, 3_{3}\right)+\widehat{\omega}_{k}\left(3_{2}, 3_{3}\right) \text {; }
$$

(viii) $\widehat{S}_{k}\left(3_{1}, 3_{2}\right)=\widehat{S_{k}}\left(3_{1}^{C}, 3_{2}^{C}\right)$;

where $k=1,2,3$.

5. The corrected Eq. 21 is given below:

To prove these properties, first, we divide $\mathfrak{U}$ into two parts $\mathfrak{U}_{\mathbf{\Delta}}$ and $\mathfrak{U}_{\mathbf{V}}$, such that

$$
\begin{aligned}
& \mathfrak{H}_{\mathbf{\Delta}}=\left\{u_{j} \mid u_{j} \in \mathfrak{U}, \mathfrak{3}_{1} \subseteq \mathfrak{3}_{2}\right\} \\
& \quad \text { and } \mathfrak{U}_{\mathbf{v}}=\left\{u_{j} \mid u_{j} \in \mathfrak{U}, \mathfrak{3}_{1} \supseteq \mathfrak{3}_{2}\right\}
\end{aligned}
$$

Note that $\forall u_{j} \in \mathfrak{H}_{\mathbf{\Delta}}$

$$
\ell_{\xi_{3_{1}}}\left(u_{j}\right) \leq \ell_{\xi_{3_{2}}}\left(u_{j}\right), \ell_{\eta_{3_{1}}}\left(u_{j}\right) \geq \ell_{\eta_{3_{2}}}\left(u_{j}\right)
$$

and also $\forall u_{j} \in \mathfrak{U}_{\mathbf{v}}$,

$$
\ell_{\xi_{3_{1}}}\left(u_{j}\right) \geq \ell_{\xi_{3_{2}}}\left(u_{j}\right), \ell_{\eta_{3_{1}}}\left(u_{j}\right) \leq \ell_{\eta_{3_{2}}}\left(u_{j}\right) \text {. }
$$

6. The corrected Sect. 4.1 Problem formulation is given below.

\section{Problem formulation}

A general MADM problem with $\mathrm{L}^{q} \mathrm{ROPF}$ information can be formulated as follows: 
Let $3=\left\{3_{1}, 3_{2}, \ldots, 3_{m}\right\}$ be a finite set of alternatives and $\mathfrak{Q}=\left\{\mathfrak{U}_{1}, \mathfrak{N}_{2}, \ldots, \mathfrak{\mathfrak { A }}_{n}\right\}$ be a set of attributes with the corresponding weight vector $\omega=\left(\omega_{1}, \omega_{2}, \ldots, \omega_{n}\right)$ satisfying with $\omega_{j} \geq 0(j=1,2, \ldots, n)$ and $\sum_{j=1}^{n} \omega_{j}=1$. Suppose that the expert evaluates the available alternatives concerning the set attributes and provides his/her evaluation information in the form of following $\mathrm{L}^{q}$ ROPFSs:

$$
\boldsymbol{3}_{i}=\left\{\left\langle\mathfrak{A}_{\mathrm{i}}, \ell_{\xi_{3_{i}}\left(\mathfrak{A}_{j}\right)}, \ell_{\eta_{3_{i}}\left(\mathfrak{A}_{j}\right)}\right\rangle \mid \mathfrak{A}_{j} \in \mathfrak{A}\right\},
$$

where $\ell_{\xi_{3 i}\left(\mathfrak{I}_{j}\right)}$ indicates the degree to which the alternative $3_{i}$ satisfies the attribute $\mathfrak{A}_{j}$ and $\ell_{\eta_{3_{i}}\left(\mathfrak{S}_{j}\right)}$ represents the degree to with the alternative $\boldsymbol{3}_{i}$ does not satisfy the attribute $\mathfrak{A}_{j}$. Here, the main objective of expert is to find the best alternative among the available alternatives.

7. The corrected Step 4 under section 4.2 A novel MADM method is given below.

Step 4: Compute the satisfaction degree $\mathfrak{R}_{i}$ corresponding to each alternative $\boldsymbol{Z}_{i}$ over the attribute set $\mathfrak{A}$ by using the following expression

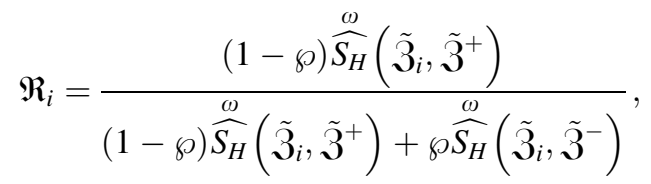

where $\wp$ represents the risk preferences of the expert. The value of the $\wp$ is provided by the expert in advanced. We can analyze the following three special cases based on the values of the $\wp$ :
Example 5 Let us consider a Venture Capital (VC) investment decision problem. Suppose a venture capital company intends to invest in an enterprise project. After making the proper market analysis, venture capital company short-lists five alternative companies for further selection: (i) An artificial intelligence company $3_{1}$ (ii) A renewable energy production company $3_{2}$ (iii) An environmental technology company $3_{3}$ (iv) A public transport management company 34 and (v) An E-sports company 35 . In order to select the best project for this investment and to generate maximum revenue, the company forms a panel of experts to conduct a comprehensive evaluation of these projects. The experts conduct a group discussion and decide the following five attributes to evaluate the available alternative projects: (i) Innovation ability $\mathfrak{A}_{1}$ (ii) Project profitability $\mathfrak{A}_{2}$ (iii) Management team quality $\mathfrak{A}_{3}$ (iv) Risk control capabilities $\mathfrak{A}_{4}(\mathrm{v})$ The competitive power in the market $\mathfrak{Q}_{5}$. Let $\omega=(0.15,0.20,0.35,0.10,0.20)^{T}$ be the weighting vector corresponding to attributes $\mathfrak{A}_{j}$. Assume that the panel of experts evaluates the available alternatives considering the attributes $\mathfrak{A}_{j}$ and provides the assessment information based on the LTS:

$\widehat{\mathbb{Q}}=\left\{\begin{array}{c}\ell_{0}=\text { extremely low }(\mathrm{EL}), \ell_{1}=\text { very low }(\mathrm{VD}), \ell_{2}=\text { low }(\mathrm{L}), \ell_{3}=\text { slightly low }(\mathrm{SL}), \\ \ell_{4}=\text { general }(\mathrm{G}), \ell_{5}=\text { slightly high }(\mathrm{SH}), \ell_{6}=\operatorname{high}(\mathrm{H}), \ell_{7}=\text { very high }(\mathrm{VH}), \\ \ell_{8}=\text { extremely high }(\mathrm{EH})\end{array}\right\}$

i $\wp<0.5$ : It indicates that the expert is pessimist.

ii $\wp>0.5$ : It means that the expert is optimist.

iii $\wp=0.5$ : It shows the neutral nature of the expert towards the result.

8. The corrected Tables $8,9,10$ and 11 are provided below:

9. The corrected Sect. 4.3 Illustrative example should read as:

\section{Illustrative example}

In this section, the developed method has been illustrative by a problem of venture capital investment with $\mathrm{L}^{q} \mathrm{ROPF}$ information.

and represents in the form of following $\mathrm{L}^{q} \mathrm{ROPFSs}$ : 
Table 8 Values of the satisfaction degree $\mathfrak{R}_{i}$ obtained by the WGHL $^{q}$ ROPFS measure

Satisfaction degree when $\alpha=1, \beta=0, \gamma=0$

$\Phi^{*}=\Phi_{1}^{*}$

\begin{tabular}{llllll}
\hline & $\mathfrak{R}_{1}$ & $\mathfrak{R}_{2}$ & $\mathfrak{R}_{3}$ & $\mathfrak{R}_{4}$ & $\mathfrak{R}_{5}$ \\
\hline$\lambda=1$ & 0.4934 & 0.5404 & 0.5584 & 0.5414 & 0.4224 \\
$\lambda=2$ & 0.5005 & 0.5419 & 0.5420 & 0.5343 & 0.4317 \\
$\lambda=5$ & 0.4999 & 0.5417 & 0.5434 & 0.5349 & 0.4311 \\
$\lambda=7$ & 0.4987 & 0.5415 & 0.5458 & 0.5362 & 0.4299 \\
$\lambda=10$ & 0.4975 & 0.5412 & 0.5486 & 0.5373 & 0.4286 \\
\hline
\end{tabular}

$\Phi^{*}=\Phi_{2}^{*}(\varrho=1.5)$

\begin{tabular}{llllll}
\hline & $\mathfrak{R}_{1}$ & $\mathfrak{R}_{2}$ & $\mathfrak{R}_{3}$ & $\mathfrak{R}_{4}$ & $\mathfrak{R}_{5}$ \\
\hline$\lambda=1$ & 0.5003 & 0.5191 & 0.5223 & 0.5180 & 0.4635 \\
$\lambda=2$ & 0.5033 & 0.5188 & 0.5162 & 0.5147 & 0.4688 \\
$\lambda=5$ & 0.5030 & 0.5188 & 0.5167 & 0.5150 & 0.4684 \\
$\lambda=7$ & 0.5024 & 0.5189 & 0.5175 & 0.5154 & 0.4677 \\
$\lambda=10$ & 0.5019 & 0.5190 & 0.5185 & 0.5159 & 0.4669
\end{tabular}

$\Phi^{*}=\Phi_{3}^{*}(\rho=\tau=0.8)$

\begin{tabular}{llllll}
\hline & $\mathfrak{R}_{1}$ & $\mathfrak{R}_{2}$ & $\mathfrak{R}_{3}$ & $\mathfrak{R}_{4}$ & $\mathfrak{R}_{5}$ \\
\hline$\lambda=1$ & 0.4836 & 0.5526 & 0.5793 & 0.5532 & 0.4045 \\
$\lambda=2$ & 0.4921 & 0.5533 & 0.5569 & 0.5427 & 0.4143 \\
$\lambda=5$ & 0.4912 & 0.5532 & 0.5588 & 0.5437 & 0.4137 \\
$\lambda=7$ & 0.4896 & 0.5529 & 0.5623 & 0.5454 & 0.4125 \\
$\lambda=10$ & 0.4880 & 0.5527 & 0.5661 & 0.5472 & 0.4111
\end{tabular}

$$
\begin{aligned}
& \mathfrak{3}_{1}=\left\{\left\langle\mathfrak{A}_{1}, \ell_{7}, \ell_{4}\right\rangle,\left\langle\mathfrak{A}_{2}, \ell_{2}, \ell_{5}\right\rangle,\left\langle\mathfrak{H}_{3}, \ell_{6}, \ell_{6}\right\rangle,\right. \\
& \left.\left\langle\mathfrak{U}_{4}, \ell_{3}, \ell_{1}\right\rangle,\left\langle\mathfrak{A}_{5}, \ell_{2}, \ell_{3}\right\rangle\right\} ; \\
& \mathfrak{3}_{2}=\left\{\left\langle\mathfrak{A}_{1}, \ell_{5}, \ell_{3}\right\rangle,\left\langle\mathfrak{U}_{2}, \ell_{7}, \ell_{2}\right\rangle,\left\langle\mathfrak{H}_{3}, \ell_{5}, \ell_{1}\right\rangle,\right. \\
& \left.\left\langle\mathfrak{A}_{4}, \ell_{3}, \ell_{6}\right\rangle,\left\langle\mathfrak{A}_{5}, \ell_{6}, \ell_{5}\right\rangle\right\} ; \\
& 3_{3}=\left\{\left\langle\mathfrak{A}_{1}, \ell_{6}, \ell_{3}\right\rangle,\left\langle\mathfrak{A}_{2}, \ell_{5}, \ell_{1}\right\rangle,\left\langle\mathfrak{N}_{3}, \ell_{6}, \ell_{3}\right\rangle,\right. \\
& \left.\left\langle\mathfrak{H}_{4}, \ell_{4}, \ell_{1}\right\rangle,\left\langle\mathfrak{A}_{5}, \ell_{4}, \ell_{3}\right\rangle\right\} ; \\
& 3_{4}=\left\{\left\langle\mathfrak{A}_{1}, \ell_{7}, \ell_{2}\right\rangle,\left\langle\mathfrak{U}_{2}, \ell_{3}, \ell_{4}\right\rangle,\left\langle\mathfrak{N}_{3}, \ell_{6}, \ell_{2}\right\rangle,\right. \\
& \left.\left\langle\mathfrak{U}_{4}, \ell_{5}, \ell_{1}\right\rangle,\left\langle\mathfrak{A}_{5}, \ell_{6}, \ell_{5}\right\rangle\right\} \\
& 3_{5}=\left\{\left\langle\mathfrak{N}_{1}, \ell_{5}, \ell_{4}\right\rangle,\left\langle\mathfrak{N}_{2}, \ell_{3}, \ell_{7}\right\rangle,\left\langle\mathfrak{A}_{3}, \ell_{6}, \ell_{6}\right\rangle,\right. \\
& \left.\left\langle\mathfrak{U}_{4}, \ell_{5}, \ell_{2}\right\rangle,\left\langle\mathfrak{U}_{5}, \ell_{2}, \ell_{7}\right\rangle\right\}
\end{aligned}
$$

\begin{tabular}{|c|c|c|c|c|c|}
\hline \multicolumn{6}{|c|}{ Satisfaction degree when $\alpha=0, \beta=1, \gamma=0$} \\
\hline \multicolumn{6}{|c|}{$\Phi^{*}=\Phi_{1}^{*}$} \\
\hline & $\mathfrak{R}_{1}$ & $\mathfrak{R}_{2}$ & $\mathfrak{R}_{3}$ & $\mathfrak{R}_{4}$ & $\mathfrak{R}_{5}$ \\
\hline$\lambda=1$ & 0.4939 & 0.5469 & 0.5728 & 0.5745 & 0.3992 \\
\hline$\lambda=2$ & 0.4998 & 0.5484 & 0.5559 & 0.5656 & 0.4077 \\
\hline$\lambda=5$ & 0.4993 & 0.5483 & 0.5574 & 0.5664 & 0.4071 \\
\hline$\lambda=7$ & 0.4983 & 0.5479 & 0.5601 & 0.5680 & 0.4060 \\
\hline$\lambda=10$ & 0.4972 & 0.5477 & 0.5629 & 0.5695 & 0.4048 \\
\hline \multicolumn{6}{|c|}{$\Phi^{*}=\Phi_{2}^{*}(\varrho=1.5)$} \\
\hline & $\mathfrak{R}_{1}$ & $\mathfrak{R}_{2}$ & $\mathfrak{R}_{3}$ & $\mathfrak{R}_{4}$ & $\mathfrak{R}_{5}$ \\
\hline$\lambda=1$ & 0.5005 & 0.5292 & 0.5333 & 0.5440 & 0.4373 \\
\hline$\lambda=2$ & 0.5036 & 0.5289 & 0.5257 & 0.5383 & 0.4431 \\
\hline$\lambda=5$ & 0.5033 & 0.5290 & 0.5264 & 0.5388 & 0.4427 \\
\hline$\lambda=7$ & 0.5028 & 0.5290 & 0.5275 & 0.5397 & 0.4418 \\
\hline$\lambda=10$ & 0.5022 & 0.5291 & 0.5288 & 0.5406 & 0.4409 \\
\hline \multicolumn{6}{|c|}{$\Phi^{*}=\Phi_{3}^{*}(\rho=\tau=0.8)$} \\
\hline & $\mathfrak{R}_{1}$ & $\mathfrak{R}_{2}$ & $\mathfrak{R}_{3}$ & $\mathfrak{R}_{4}$ & $\mathfrak{R}_{5}$ \\
\hline$\lambda=1$ & 0.4831 & 0.5572 & 0.5937 & 0.5870 & 0.3828 \\
\hline$\lambda=2$ & 0.4908 & 0.5579 & 0.5717 & 0.5751 & 0.3918 \\
\hline$\lambda=5$ & 0.4899 & 0.5578 & 0.5737 & 0.5763 & 0.3913 \\
\hline$\lambda=7$ & 0.4886 & 0.5575 & 0.5772 & 0.5783 & 0.3902 \\
\hline$\lambda=10$ & 0.4872 & 0.5573 & 0.5810 & 0.5803 & 0.3888 \\
\hline
\end{tabular}

Table 9 Values of the satisfaction degree $\mathfrak{R}_{i}$ obtained by the WGHL ${ }^{q}$ ROPFS measure

Based the information, the decision steps of developed method are executed as follows:

Step 1: Since all the attributes are benefit type, so their values need not be normalized. Hence $\tilde{3}_{i}=3_{i}$.

Step 2: We obtain the positive ideal solution $\tilde{3}^{+}$and the negative ideal solution $\tilde{\mathfrak{Z}}^{-}$given as:

$$
\begin{aligned}
& \tilde{\mathfrak{Z}}^{+}=\left\{\left\langle\mathfrak{U}_{1}, \ell_{7}, \ell_{2}\right\rangle,\left\langle\mathfrak{H}_{2}, \ell_{7}, \ell_{1}\right\rangle,\left\langle\mathfrak{H}_{3}, \ell_{6}, \ell_{1}\right\rangle,\right. \\
& \left.\left\langle\mathfrak{H}_{4}, \ell_{5}, \ell_{1}\right\rangle,\left\langle\mathfrak{H}_{5}, \ell_{6}, \ell_{3}\right\rangle\right\}, \\
& \tilde{\mathfrak{3}}^{-}=\left\{\left\langle\mathfrak{H}_{1}, \ell_{5}, \ell_{4}\right\rangle,\left\langle\mathfrak{H}_{2}, \ell_{2}, \ell_{7}\right\rangle,\left\langle\mathfrak{A}_{3}, \ell_{5}, \ell_{6}\right\rangle,\right. \\
& \left.\left\langle\mathfrak{H}_{4}, \ell_{3}, \ell_{6}\right\rangle,\left\langle\mathfrak{H}_{5}, \ell_{2}, \ell_{7}\right\rangle\right\} .
\end{aligned}
$$


Table 10 Values of the satisfaction degree $\mathfrak{R}_{i}$ obtained by the WGHL $^{q}$ ROPFS measure

Satisfaction degree when $\alpha=0, \beta=0, \gamma=1$

\begin{tabular}{llllll}
\hline$\Phi^{*}=\Phi_{1}^{*}$ & & & & & \\
\hline & $\mathfrak{R}_{1}$ & $\mathfrak{R}_{2}$ & $\mathfrak{R}_{3}$ & $\mathfrak{R}_{4}$ & $\mathfrak{R}_{5}$ \\
\hline$\lambda=1$ & 0.4923 & 0.5663 & 0.6090 & 0.6308 & 0.3491 \\
$\lambda=2$ & 0.4988 & 0.5682 & 0.5868 & 0.6162 & 0.3590 \\
$\lambda=5$ & 0.4982 & 0.5680 & 0.5888 & 0.6176 & 0.3583 \\
$\lambda=7$ & 0.4970 & 0.5676 & 0.5926 & 0.6201 & 0.3570 \\
$\lambda=10$ & 0.4957 & 0.5672 & 0.5964 & 0.6225 & 0.3555 \\
\hline
\end{tabular}

$\Phi^{*}=\Phi_{2}^{*}(\varrho=1.5)$

\begin{tabular}{llllll}
\hline & $\mathfrak{R}_{1}$ & $\mathfrak{R}_{2}$ & $\mathfrak{R}_{3}$ & $\mathfrak{R}_{4}$ & $\mathfrak{R}_{5}$ \\
\hline$\lambda=1$ & 0.5010 & 0.5463 & 0.5532 & 0.5807 & 0.3967 \\
$\lambda=2$ & 0.5050 & 0.5458 & 0.5418 & 0.5705 & 0.4046 \\
$\lambda=5$ & 0.5046 & 0.5457 & 0.5428 & 0.5714 & 0.4040 \\
$\lambda=7$ & 0.5039 & 0.5460 & 0.5447 & 0.5729 & 0.4028 \\
$\lambda=10$ & 0.5031 & 0.5461 & 0.5466 & 0.5746 & 0.4015 \\
\hline
\end{tabular}

$\Phi^{*}=\Phi_{3}^{*}(\rho=\tau=0.8)$

\begin{tabular}{llllll}
\hline & $\mathfrak{R}_{1}$ & $\mathfrak{R}_{2}$ & $\mathfrak{R}_{3}$ & $\mathfrak{R}_{4}$ & $\mathfrak{R}_{5}$ \\
\hline$\lambda=1$ & 0.4770 & 0.5777 & 0.6362 & 0.6498 & 0.3304 \\
$\lambda=2$ & 0.4851 & 0.5784 & 0.6087 & 0.6315 & 0.3405 \\
$\lambda=5$ & 0.4842 & 0.5782 & 0.6113 & 0.6332 & 0.3398 \\
$\lambda=7$ & 0.4827 & 0.5779 & 0.6159 & 0.6362 & 0.3385 \\
$\lambda=10$ & 0.4811 & 0.5776 & 0.6208 & 0.6392 & 0.3370 \\
& & & & &
\end{tabular}

Step 3: We calculate the degree of similarity between each $\tilde{\mathfrak{Z}}_{i}$ and $\tilde{\mathfrak{Z}}^{+}$; and the degree of similarity between each $\tilde{\mathfrak{Z}}_{i}$ and $\tilde{\mathfrak{Z}}^{-}$, respectively, based on Eqs. (41) and (42). The obtained values are summarized in Tables 4 to 7 . (Here, we have taken $q=3$ )

Step 4: Utilizing the Eq. (43), we calculate the satisfaction degree $\mathfrak{R}_{i}$ for each alternative, and the resulted values are recorded in Tables 8 to 11 . Without loss of generality, we consider $\wp=0.5$.
Table 11 Values of the satisfaction degree $\mathfrak{R}_{i}$ obtained by the WGHL ${ }^{q}$ ROPFS measure

\begin{tabular}{|c|c|c|c|c|c|}
\hline \multicolumn{6}{|c|}{ Satisfaction degree when $\alpha=0.33, \beta=0.33, \gamma=0.33$} \\
\hline \multicolumn{6}{|l|}{$\Phi^{*}=\Phi_{1}^{*}$} \\
\hline & $\mathfrak{R}_{1}$ & $\mathfrak{R}_{2}$ & $\mathfrak{R}_{3}$ & $\mathfrak{R}_{4}$ & $\mathfrak{R}_{5}$ \\
\hline$\lambda=1$ & 0.4935 & 0.5500 & 0.5773 & 0.5782 & 0.3933 \\
\hline$\lambda=2$ & 0.4999 & 0.5516 & 0.5592 & 0.5684 & 0.4024 \\
\hline$\lambda=5$ & 0.4992 & 0.5514 & 0.5608 & 0.5694 & 0.4018 \\
\hline$\lambda=7$ & 0.4981 & 0.5511 & 0.5637 & 0.5710 & 0.4006 \\
\hline$\lambda=10$ & 0.4969 & 0.5508 & 0.5668 & 0.5726 & 0.3993 \\
\hline \multicolumn{6}{|c|}{$\Phi^{*}=\Phi_{2}^{*}(\varrho=1.5)$} \\
\hline & $\mathfrak{R}_{1}$ & $\mathfrak{R}_{2}$ & $\mathfrak{R}_{3}$ & $\mathfrak{R}_{4}$ & $\mathfrak{R}_{5}$ \\
\hline$\lambda=1$ & 0.5006 & 0.5305 & 0.5350 & 0.5452 & 0.4348 \\
\hline$\lambda=2$ & 0.5039 & 0.5302 & 0.5269 & 0.5392 & 0.4409 \\
\hline$\lambda=5$ & 0.5036 & 0.5303 & 0.5275 & 0.5397 & 0.4405 \\
\hline$\lambda=7$ & 0.5030 & 0.5303 & 0.5288 & 0.5406 & 0.4396 \\
\hline$\lambda=10$ & 0.5024 & 0.5304 & 0.5301 & 0.5415 & 0.4387 \\
\hline \multicolumn{6}{|c|}{$\Phi^{*}=\Phi_{3}^{*}(\rho=\tau=0.8)$} \\
\hline & $\mathfrak{R}_{1}$ & $\mathfrak{R}_{2}$ & $\mathfrak{R}_{3}$ & $\mathfrak{R}_{4}$ & $\mathfrak{R}_{5}$ \\
\hline$\lambda=1$ & 0.4817 & 0.5612 & 0.5999 & 0.5922 & 0.3756 \\
\hline$\lambda=2$ & 0.4898 & 0.5620 & 0.5763 & 0.5790 & 0.3853 \\
\hline$\lambda=5$ & 0.4890 & 0.5618 & 0.5784 & 0.5801 & 0.3846 \\
\hline$\lambda=7$ & 0.4875 & 0.5615 & 0.5822 & 0.5824 & 0.3835 \\
\hline$\lambda=10$ & 0.4860 & 0.5613 & 0.5862 & 0.5846 & 0.3820 \\
\hline
\end{tabular}

Step 5. We rank all the alternatives $\boldsymbol{B}_{i}(i=1,2,3,4,5)$ based on the values of satisfaction degree in descending order. Table 12 presents the ranking results of the alternatives.

From Table 12, we see that the ranking order of alternatives changes slightly as the parameters value change. For $\alpha=1, \beta=0$ and $\gamma=0$, the alternative $3_{3}$ is the best alternative when $\Phi^{*}=\Phi_{1}^{*}$ and $\Phi_{3}^{*}$, whereas $3_{2}$ becomes the best alternative when $\Phi^{*}=\Phi_{2}^{*}$. Besides, if we take $\alpha=$ $0, \beta=1, \gamma=0 \quad$ or $\quad \alpha=0, \beta=0, \gamma=1 \quad$ or $\alpha=\beta=1=\gamma=0.33$, then the alternative $3_{4}$ obtains as a 
Table 12 Ranking order of the alternatives based the WGHL $^{q}$ ROPFS measure

\begin{tabular}{|c|c|c|c|}
\hline \multicolumn{4}{|c|}{ Ranking order } \\
\hline \multicolumn{4}{|c|}{$\alpha=1, \beta=0, \gamma=0$} \\
\hline & $\Phi^{*}=\Phi_{1}^{*}$ & $\Phi^{*}=\Phi_{2}^{*}(\varrho=1.5)$ & $\Phi^{*}=\Phi_{3}^{*}(\rho=\tau=0.8)$ \\
\hline$\lambda_{1}$ & $3_{3} \succ 3_{4} \succ 3_{2} \succ 3_{1} \succ 3_{5}$ & $3_{3} \succ 3_{2} \succ 3_{4} \succ 3_{1} \succ 3_{5}$ & $3_{3} \succ 3_{4} \succ 3_{2} \succ 3_{1} \succ 3_{5}$ \\
\hline$\lambda_{2}$ & $3_{3} \succ 3_{2} \succ 3_{4} \succ 3_{1} \succ 3_{5}$ & $3_{2} \succ 3_{3} \succ 3_{4} \succ 3_{1} \succ 3_{5}$ & $3_{3} \succ 3_{2} \succ 3_{4} \succ 3_{1} \succ 3_{5}$ \\
\hline$\lambda_{5}$ & $3_{3} \succ 3_{2} \succ 3_{4} \succ 3_{1} \succ 3_{5}$ & $3_{2} \succ 3_{3} \succ 3_{4} \succ 3_{1} \succ 3_{5}$ & $3_{3} \succ 3_{2} \succ 3_{4} \succ 3_{1} \succ 3_{5}$ \\
\hline$\lambda_{7}$ & $3_{3} \succ 3_{2} \succ 3_{4} \succ 3_{1} \succ 3_{5}$ & $3_{2} \succ 3_{3} \succ 3_{4} \succ 3_{1} \succ 3_{5}$ & $3_{3} \succ 3_{2} \succ 3_{4} \succ 3_{1} \succ 3_{5}$ \\
\hline$\lambda_{10}$ & $3_{3} \succ 3_{2} \succ 3_{4} \succ 3_{1} \succ 35$ & $3_{2} \succ 3_{3} \succ 3_{4} \succ 3_{1} \succ 3_{5}$ & $3_{3} \succ 3_{2} \succ 3_{4} \succ 3_{1} \succ 3_{5}$ \\
\hline
\end{tabular}

$\alpha=0, \beta=1, \gamma=0$

\begin{tabular}{llll}
\hline & $\Phi^{*}=\Phi_{1}^{*}$ & $\Phi^{*}=\Phi_{2}^{*}(\varrho=1.5)$ & $\Phi^{*}=\Phi_{3}^{*}(\rho=\tau=0.8)$ \\
\hline$\lambda_{1}$ & $3_{4} \succ 3_{3} \succ 3_{2} \succ 3_{1} \succ 3_{5}$ & $3_{4} \succ 3_{3} \succ 3_{2} \succ 3_{1} \succ 3_{5}$ & $3_{3} \succ 3_{4} \succ 3_{2} \succ 3_{1} \succ 3_{5}$ \\
$\lambda_{2}$ & $3_{4} \succ 3_{3} \succ 3_{2} \succ 3_{1} \succ 3_{5}$ & $3_{4} \succ 3_{2} \succ 3_{3} \succ 3_{1} \succ 3_{5}$ & $3_{4} \succ 3_{3} \succ 3_{2} \succ 3_{1} \succ 3_{5}$ \\
$\lambda_{5}$ & $3_{4} \succ 3_{3} \succ 3_{2} \succ 3_{1} \succ 3_{5}$ & $3_{4} \succ 3_{2} \succ 3_{3} \succ 3_{1} \succ 3_{5}$ & $3_{4} \succ 3_{3} \succ 3_{2} \succ 3_{1} \succ 3_{5}$ \\
$\lambda_{7}$ & $3_{4} \succ 3_{3} \succ 3_{2} \succ 3_{1} \succ 3_{5}$ & $3_{4} \succ 3_{2} \succ 3_{3} \succ 3_{1} \succ 3_{5}$ & $3_{4} \succ 3_{3} \succ 3_{2} \succ 3_{1} \succ 3_{5}$ \\
$\lambda_{10}$ & $3_{4} \succ 3_{3} \succ 3_{2} \succ 3_{1} \succ 3_{5}$ & $3_{4} \succ 3_{2} \succ 3_{3} \succ 3_{1} \succ 3_{5}$ & $3_{3} \succ 3_{4} \succ 3_{2} \succ 3_{1} \succ 3_{5}$ \\
\hline
\end{tabular}

$\alpha=0, \beta=0, \gamma=1$

\begin{tabular}{llll}
\hline & $\Phi^{*}=\Phi_{1}^{*}$ & $\Phi^{*}=\Phi_{2}^{*}(\varrho=1.5)$ & $\Phi^{*}=\Phi_{3}^{*}(\rho=\tau=0.8)$ \\
\hline$\lambda_{1}$ & $3_{4} \succ 3_{3} \succ 3_{2} \succ 3_{1} \succ 3_{5}$ & $3_{4} \succ 3_{3} \succ 3_{2} \succ 3_{1} \succ 3_{5}$ & $3_{4} \succ 3_{3} \succ 3_{2} \succ 3_{1} \succ 3_{5}$ \\
$\lambda_{2}$ & $3_{4} \succ 3_{3} \succ 3_{2} \succ 3_{1} \succ 3_{5}$ & $3_{4} \succ 3_{2} \succ 3_{3} \succ 3_{1} \succ 3_{5}$ & $3_{4} \succ 3_{3} \succ 3_{2} \succ 3_{1} \succ 3_{5}$ \\
$\lambda_{5}$ & $3_{4} \succ 3_{3} \succ 3_{2} \succ 3_{1} \succ 3_{5}$ & $3_{4} \succ 3_{2} \succ 3_{3} \succ 3_{1} \succ 3_{5}$ & $3_{4} \succ 3_{3} \succ 3_{2} \succ 3_{1} \succ 3_{5}$ \\
$\lambda_{7}$ & $3_{4} \succ 3_{3} \succ 3_{2} \succ 3_{1} \succ 3_{5}$ & $3_{4} \succ 3_{2} \succ 3_{3} \succ 3_{1} \succ 3_{5}$ & $3_{4} \succ 3_{3} \succ 3_{2} \succ 3_{1} \succ 3_{5}$ \\
$\lambda_{10}$ & $3_{4} \succ 3_{3} \succ 3_{2} \succ 3_{1} \succ 3_{5}$ & $3_{4} \succ 3_{3} \succ 3_{2} \succ 3_{1} \succ 3_{5}$ & $3_{4} \succ 3_{3} \succ 3_{2} \succ 3_{1} \succ 3_{5}$ \\
\hline
\end{tabular}

$\alpha=0.33, \beta=0.33, \gamma=0.33$

\begin{tabular}{llll}
\hline & $\Phi^{*}=\Phi_{1}^{*}$ & $\Phi^{*}=\Phi_{2}^{*}(\varrho=1.5)$ & $\Phi^{*}=\Phi_{3}^{*}(\rho=\tau=0.8)$ \\
\hline$\lambda_{1}$ & $3_{4} \succ 3_{3} \succ 3_{2} \succ 3_{1} \succ 3_{5}$ & $3_{4} \succ 3_{3} \succ 3_{2} \succ 3_{1} \succ 3_{5}$ & $3_{4} \succ 3_{3} \succ 3_{2} \succ 3_{1} \succ 3_{5}$ \\
$\lambda_{2}$ & $3_{4} \succ 3_{3} \succ 3_{2} \succ 3_{1} \succ 3_{5}$ & $3_{4} \succ 3_{2} \succ 3_{3} \succ 3_{1} \succ 3_{5}$ & $3_{4} \succ 3_{3} \succ 3_{2} \succ 3_{1} \succ 3_{5}$ \\
$\lambda_{5}$ & $3_{4} \succ 3_{3} \succ 3_{2} \succ 3_{1} \succ 3_{5}$ & $3_{4} \succ 3_{2} \succ 3_{3} \succ 3_{1} \succ 3_{5}$ & $3_{4} \succ 3_{3} \succ 3_{2} \succ 3_{1} \succ 3_{5}$ \\
$\lambda_{7}$ & $3_{4} \succ 3_{3} \succ 3_{2} \succ 3_{1} \succ 3_{5}$ & $3_{4} \succ 3_{2} \succ 3_{3} \succ 3_{1} \succ 3_{5}$ & $3_{4} \succ 3_{3} \succ 3_{2} \succ 3_{1} \succ 3_{5}$ \\
$\lambda_{9}$ & $3_{4} \succ 3_{3} \succ 3_{2} \succ 3_{1} \succ 3_{5}$ & $3_{4} \succ 3_{2} \succ 3_{3} \succ 3_{1} \succ 3_{5}$ & $3_{3} \succ 3_{4} \succ 3_{2} \succ 3_{1} \succ 3_{5}$ \\
\hline
\end{tabular}

Table 13 The score values of the alternatives based on different aggregation operators

\begin{tabular}{|c|c|c|c|c|c|c|}
\hline \multirow[t]{2}{*}{ Aggregation operator } & \multicolumn{5}{|c|}{ Score value } & \multirow[t]{2}{*}{ Ranking order } \\
\hline & $P\left(3_{1}\right)$ & $P\left(3_{2}\right)$ & $P\left(3_{3}\right)$ & $P\left(3_{4}\right)$ & $P\left(3_{5}\right)$ & \\
\hline $\mathrm{L}^{q} \mathrm{ROPFWA}$ & 6.7243 & 7.0303 & 6.9115 & 7.0419 & 6.2129 & $3_{4} \succ 3_{2} \succ 3_{3} \succ 3_{1} \succ 3_{5}$ \\
\hline $\mathrm{L}^{q} \mathrm{ROPFWG}$ & 6.0264 & 6.6916 & 6.7936 & 6.7394 & 5.4836 & $3_{3} \succ 3_{4} \succ 3_{2} \succ 3_{1} \succ 3_{5}$ \\
\hline $\mathrm{L}^{q}$ ROPFIWA & 6.3460 & 6.8791 & 6.8733 & 6.9490 & 5.8256 & $3_{4} \succ 3_{2} \succ 3_{3} \succ 3_{1} \succ 3_{5}$ \\
\hline $\mathrm{L}^{q}$ ROPFIWG & 6.6199 & 6.8502 & 6.8845 & 6.9485 & 5.7606 & $3_{4} \succ 3_{3} \succ 3_{2} \succ 3_{1} \succ 3_{5}$ \\
\hline
\end{tabular}


best alternative with all LSFs. It is worth noting that that the alternative $3_{5}$ is the worst alternative in all the ranking results.

10. The corrected Tables 12 and 13 are as follows:

The original article has been corrected.
Publisher's Note Springer Nature remains neutral with regard to jurisdictional claims in published maps and institutional affiliations. 